\title{
in \\ EXTENSIONS OF KALMAN RECURSIVE ESTIMATION THEORY TO RETROSPECTIVE UPDATING AND INTERPOLATION WITH APPLICATIONS TO INSTRUMENT CALIBRATION
}

by

C. ROGER GLASSEY

and

YOON C. CHOY

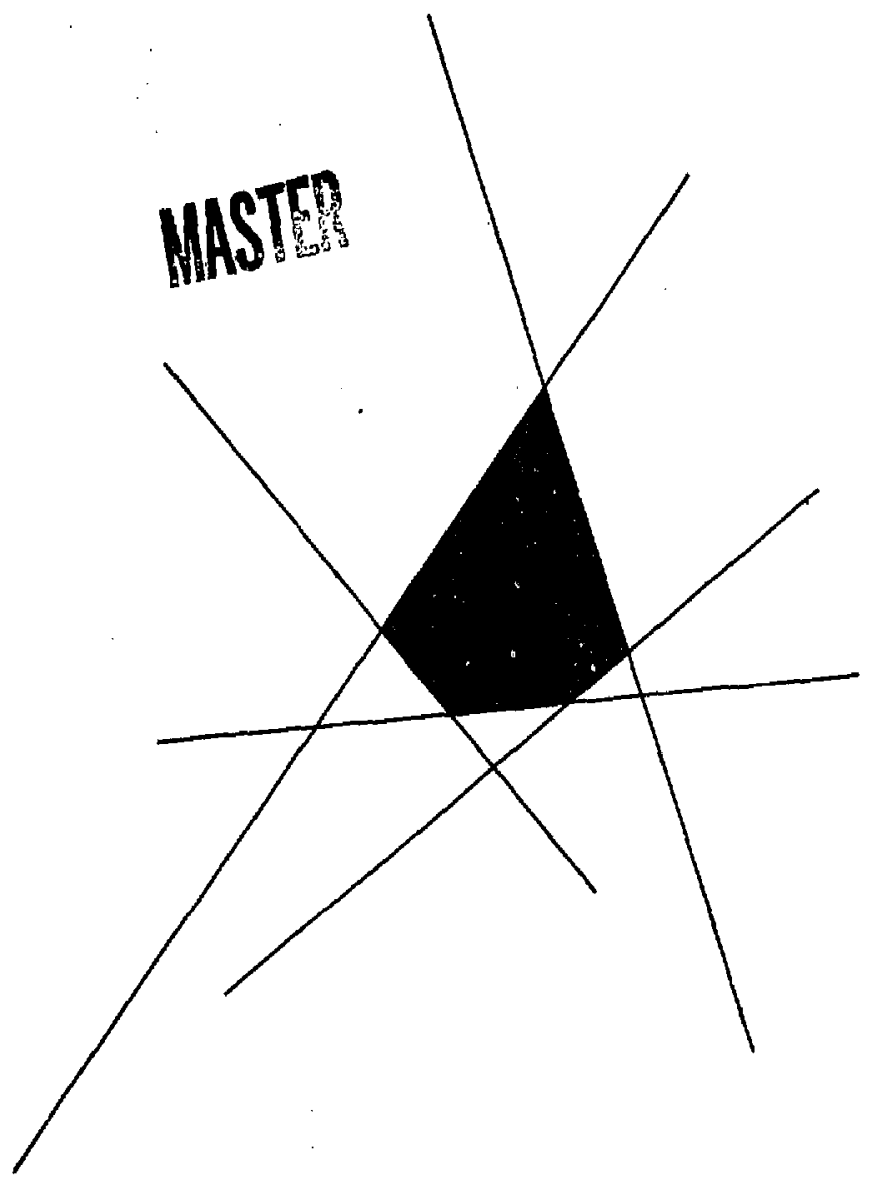

OPERATIONS

RESEARCH

CENTER

$P .0 .3684703$ 
EXTENSIONS OF KALMAN RECURSIVE ESTIMATION THEORY TO RETROSF CCTIVE UPDATING AND INTERPOLATION WITH APPLICATIONS TO INSTROMENT CALTBRATION

\author{
C. Roger Glassey \\ Department of Industrial Engineering \\ and Operations Research \\ University of California, Berkeley
}

and

Yoon C. Choy

Operations Research Center

University of Calffornia, Berkeley

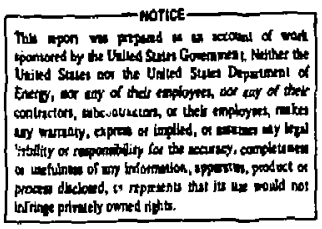

This research was supported by the Lawrence Livermore Laboratory under Purchase Order No. 3684703 with the University of Califoraia. Reproduction in whole or in part is permitted for any purpose of the United States Government. 


\section{ABSTRACT}

In this report, we consider the problem of estimation of a physical quantity from a set of measurements. We extend Kalman recursive estimation procedure in two ways. First, we explore how to use the latest observation to retrospectively update estimates of past system states. Second, we show how to apply the retrospective update 1dea to get interpolation estimates between the epochs of observations. We also show application of these ideas for instrument calibration in nuclear accountability systems. 
CHAPTER 0: INTRODUCTION AND SUMMARY ............ 1

CHAPTER 1: REVIEW OF THE THEORY OF BEST LINEAR UNBIASED ESTIMATORS AND KALMAN RECURSIVE ESTIMATION . . . . 3

1.1 Best Linear Unbiased Estimators ... . . . . . 3

1.2 Kalman Recursive Estimators ........... 4

CHAPTER 2: APPLICATION OF RECURSIVE LINEAR ESTIMATION TO INSTRIMENT CALIBRATION ..........8

CHAPTER 3: RETROSPECTIVE UPDATE ............... 13

CHAPTER 4: APPLICATION OF RETROSPECTIVE UPDATE TO INSTRUMENT CALIBRATION ............. 15

CHAPTER 5: INTERPOLATION COMBLNED WITH RETROSPECTIVE UPDATE . . . . . . . . . . 22

5.1 Update of Estimate at Observation Epochs . . . . . . 22

5.2 Interpolation ............... 24

5.2 .1 Interpolation then $\mathrm{n}=\mathrm{m}-1 \ldots 25$

5.2.2 Retrospective Update of Interpolation: $m>n+125$

5.3 summary ................... 27

CHAPTER 6: INTERPOLATION AND RETROSPECTIVE UPDATE OF INSTRUNENT CALIBRATION . . . . . . . . . . . 28

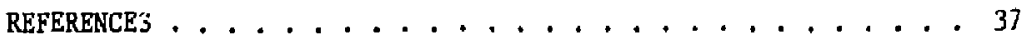


EXTENSIONS OF KALMAN RECURSIVE ESTIMATION THEORY TO RETROSPECTIVE UPDATING AND INTERPOLATION WITH APPLICATIONS TO INSTRUMENT CALIBRATION

\section{By}

C. Roger Glassey and Yoon C. Choy

\section{INTRODUCTION AND SUMMARY}

Is nuclear materials accountability systems, the requirements for precision and accuracy are quite stringent. It is therefore important to extract as much information as possible from the data that is collected by both process instrumentation and from laboratory analysis. When the data consists of a time serles of correlated observations, the correlation structure can be utilized to improve the precision of estimates computed from the data.

One of the widely used techniques for making such computatioas is due to Kalman (see Reference [1] and [2]). This Kalman recursive estimation procedure is usually employed to estimate the state of the system of interest at the time of the most recent observation. In this report, we consider two extensions of this scheme. Flrst, we explore how the latest observation can be used to retrospectively update estimates of past system states, thereby improving the precision of these estimates. Second, we consider situations in which it is desired to estimate the state of the system at times between the epochs of the observations. Finally, we show how to apply to retrospective update idea to these interpolation estimates.

All the estimators we consider are Best Linear Unbiased Estimators (BLUE) In the sense that the expected value of the estimator is the true value of the state; they are conputed by linear transformations of the data; and they have minimum variance in the class of estimators having the first two 
properties. The Kalman scheme recursively computes the BLUE of the system state and also the variance matrix of the estimator from the previous estimate, the previous variance matrix, and the latest observation. The propertles of BLJE and the Kalman scheme are reviewed in Section 1.

The deviation of the retrospective update scheme is presented in Section 3 and the interpolation scheme, in Section 5.

There are many aspects of nuclear materials accountability to which these Ideas could be applied. We have selected instrument calibration to illustrate our methods because the models are relatively simple, and because not much appears in the literature in the problem of designing a calibration strategy for an instrument to take advantage of the known (or suspected) way in which parameters drift over time.

The organization of this report is to illustrate each section of theory by applying it to the Instrument calibration problem. Thus, Section 2 deals with the Kalman update of estimated caltbration coefficlents; Section 4 with retrospective update 1.e., improving the estimate of last week's parameters based on this week's calibration results; Section 6 with interpolation i.e., how to estimate Wednesday's parameters based on calibrations performed on Monday and Friday. Section 6 also discusses circumstances under which retrospect:ive update of coefficients is worthwhile, and suggests some general ideas about calibration strategies.

The example calculations show that the precision of estimated instrument calibration coefficients can be greatly increased by the use of the extenstons to the Ralman updating scheme developed in this paper. Applications of these dieas to other areas of accountability systems may show similar potential improvements in precision. 
1. REVIEW OF THE THEORY OF BEST LINEAR UNBIASED ESTIMATORS AND KAIMAN RECURSIVE ESTIMATION

\subsection{Best Linear Unbiased Estimators}

Consider two jointly distributed vector valued random varlables, $X$ and $Y$ with mean $H_{x}$ and $\nu_{y}$ respectively. We denote the variance-covariance matrix as

$$
E\left\{\left(X-\mu_{x}\right)\left(Y-\mu_{y}\right)^{\prime}\right\}=C\{X, Y\}
$$

where $\mathbf{E}$ is the expectation operator and the prime symbol ' denotes a vector or matrix. If $X \in R_{m}$ and $Y \in R_{n}^{* *}$ then $C\{X, Y\}$ will be an In $x$ m matrix. Clearly, $C\{Y, X\}=C\{X, Y\}^{\prime}$, and the matrices $C\{X, X\}$ and $C\{\mathrm{Y}, \mathrm{Y}\}$ will be square and of order $\mathrm{n}$.

Now suppose one observes the value of the vector $X$ and desires to compute the $\hat{Y}$, Best Linear Unbiased Estimator (BLUE) of $Y$, given this observation. It is well known that

$$
\hat{\mathrm{Y}}=\mu_{\mathrm{y}}+\mathrm{BZ}
$$

where $Z=X-\mu_{X}$ and $B$ is given by

$$
B=C\{Y, X\} C\{X, X\}^{-1}
$$

Define the residual vector $R=Y-\hat{Y}$. Then $C\{R, R\}$ may be computed from

$$
C\{R, R\}=C\{Y, Y\}-B C\{X, Y\}
$$

In this paper, all vectors are column vectors unless otherwise specifled. ${ }^{* *} R_{\text {II }}$ is a real m-dimensional vector space. 
Finally, it is easy to show that

$$
C\{R, Z\}=0 \text {. }
$$

\subsection{Kalman Recursive Estimators}

Now consider the discrete-time, dynamic system given by:

$$
Y_{t+1}=A Y_{t}+V_{t}
$$

$$
x_{t}=Y_{t}+w_{t}
$$

where $Y_{t}$ is the n-dimensional state vector of the system at time $t, A$ is a known matrix of order $n$, called the state transition matrix, and $V_{t}$ is a vector valued random variable. The m-vector $X_{t}$ is observed at time $t$; the $m \times n$ matrix $M$ is known, and $W_{t}$ is an $m$ vector valued random variable. We assume that $E\left\{V_{t}\right\}$ and $E\left\{W_{t}\right\}$ are both zero vectors, and

$$
\mathrm{C}\left\{\mathrm{v}_{\mathrm{t}}, \mathrm{v}_{\mathrm{s}}\right\}=\delta(\mathrm{t}-\mathrm{s}) \mathrm{C}_{\mathrm{v}}
$$

$$
\mathrm{C}\left\{\mathrm{W}_{t}, \mathrm{~W}_{\mathrm{s}}\right\}=\delta(t-\mathrm{s}) \mathrm{C}_{\mathrm{W}}
$$

$$
\mathrm{C}\left\{\mathrm{v}_{\mathrm{t}}, \mathrm{W}_{\mathrm{s}}\right\}=0 \text { for all } \mathrm{t} \text { and } \mathrm{s}
$$

where the covariance matrices $C_{v}$ and $C_{w}$ are known and the delta function $\delta(t)=1$ when $t=0$ and is zero otherwise. 
Thus the sequences $V_{t}$ and $W_{t}$ are mutually and temporally uncorrelated; $V_{t}$ may be regarded as a random perturbation of the state vector, and $W_{t}$ as representing measurement error.

\section{Definitions:}

$\hat{Y}_{t \mid s}=$ BLUE of $Y_{t}$ given history of observations up to $X_{s}$

$\hat{x}_{t \mid s}=$ BLUE of $x_{t}$ given the same history. Note, when $s \geq t$, $x_{t}$ has been observed so $\hat{x}_{t \mid s}=x_{t}$

$R_{t \mid s}=Y_{t}-\hat{Y}_{t \mid s}$

$c\left[z_{t}\right]=E\left\{\left(x_{t}-\hat{x}_{t \mid t-1}\right)\left(x_{t}-\hat{x}_{t \mid t-1}\right) !\right.$

$C\left[R_{t \mid s}\right]=C\left\{R_{t \mid s}, R_{t \mid s}\right\}$.

The Kalman scheme (see Reference [1]) is a method of recursively computing $\hat{Y}_{t+1 \mid t}$. Assume we have computed $\hat{Y}_{t \mid t-1}$, the BLUE of $Y_{t}$ given the observation history up to $x_{t-1}$. Then we observe $x_{t}$ and desire to update both oilr estimate of $Y_{t}$ and, extrapolating one period into the future, compute the BLUE of $\mathrm{Y}_{t+1}$. To obtain $\hat{Y}_{t \mid t}$, we use Equation (1.2) and (1.3). Since $\hat{Y}_{t / t-1}$ is the BLUE of $Y_{t}$, It is the conditional expectation of $Y_{t}$ before $X_{t}$ is observed; hence it plays the role of $\mu_{y}$ in (1.1). Similarly, $\hat{x}_{t \mid t-1}$ is the conditional expectation of $x_{t}$ given the nistory to $t-1$ and substitutes for $\mu_{x}$. Since $x_{t}$ is linearly related to $Y_{t}$ by $(1.8), \hat{X}_{t / t}$ is given by

$$
\hat{\mathrm{X}}_{t \mid t-1}=\hat{\mathrm{Y}}_{t \mid t-1} \text {. }
$$


We also need the conditional variance matrix $C\left[z_{t}\right]$, which can be computed from its definition as follows: subtract (1.10) from (1.8) and use the definition of $R_{t \mid t}$ to obtain

$$
z_{t}=x_{t}-\hat{x}_{t \mid t-1}=\mathbb{R}_{t \mid t-1}+W_{t}
$$

Hence, since $\hat{x}_{t \mid t-1}$ is the conditional expectation of $x_{t}^{\prime}$,

$$
\begin{aligned}
C\left[Z_{t}\right] & =E\left\{\left(M R_{t \mid t-1}+W_{t}\right)\left(M R_{t \mid t-1}+W_{t}\right)^{\prime}\right\} \\
& =M C\left[R_{t \mid t-1}\right] M^{\prime}+C_{w} .
\end{aligned}
$$

In this computation, the cross product terms (covariance of $R_{t \mid t-1}$ with $W_{t}$ ) vanish because $R_{t} \mid t-1$ depends upon the $V_{t}$ sequence and the $W_{k}$ sequence for values of $k$ less than $t$; but $W_{t}$ is uncorrelated with both. Similarly, we compute

(1.13) $\quad C\left\{z_{t}, R_{t \mid t-1}\right\}=E\left\{\left(\mathbb{R} R_{t \mid t-1}+w_{t}\right)\left(R_{t \mid t-1}\right)^{\prime}\right\}=M C\left[R_{t ! t-1}\right]$

and, using (1.12),

$$
B_{t \mid t}=C\left[R_{t \mid t-1}\right] M^{\prime}\left(M C\left[R_{t \mid t-1}\right] M^{\prime}+C_{w}\right)^{-1}
$$

After observing the measurement $X_{t}$, we can update our estimate of $Y_{t}$ from

$$
\hat{Y}_{t \mid t}=\hat{Y}_{t \mid t-1}+B_{t \mid t}\left(X_{t}-\hat{Y Y}_{t \mid t-1}\right)
$$

which is an application of (1.1). 
The varlance matrix of $\left.R_{t}\right|_{t}$ can be computed by applying (1.5) and using (1.13) to obtain:

$$
C\left[R_{t \mid t}\right]=C\left[R_{t \mid t-1}\right]-B_{t \mid t} M C\left[R_{t \mid t-1}\right]
$$

To carry out the extrapolation, we use $(1.7)$ to conclude that

$$
\hat{Y}_{t+l \mid t}=A \hat{Y}_{t \mid t}
$$

and

$$
R_{t+1] t}=Y_{t+1}-\hat{Y}_{t+1 \mid t}=A Y_{t}-A \hat{Y}_{t \mid t}+V_{t}
$$

$$
\begin{gathered}
R_{t+1 \mid t}=A R_{t \mid t}+V_{t} \\
C\left[R_{t+1 \mid t}\right]=A C\left[R_{t \mid t}\right] A^{\prime}+C_{v} .
\end{gathered}
$$

Again, the covariance of $\left.R_{t}\right|_{t}$ with $V_{t}$ j.s zero because $R_{t / t}$ depends only on $v_{t-1}$, $W_{t}$ and older random variables which are uncorrelated with $V_{t}$, Finally, we observe that, from Equation (1.6), $C\left\{R_{t} \mid t, z_{t}\right\}=0$. Then, by dicect substitution of Equation (1.7), (1.8), and (1.17a) into (1.15) and by using the definition of $R_{t / t}$ we obtain

$$
R_{t \mid t}=\left(I-B_{t \mid t} M\right) A R_{t-1 \mid t-1}+\left(I-B_{t}\left|t^{M) V_{t-1}}-B_{t}\right| t_{t} W\right.
$$

Hence, by induction,

$$
C\left\{R_{t \mid t}, z_{s}\right\}=0 \text { for all } s \leq t
$$

Thus the residual at time $t$ is uncorrelated with the history of the process preceeding $t$. 


\section{APPLICACION OF RECURSIVE LINEAR ESTIMATION TO INSTRUMENT CALIBRATION}

One of the applications of recursive estimacion to nuclear materials accountability is to the problem of laboracory instrument calibration. Since it is vital to obtain very precise est.mates of concentration and isotopic composition of samples taken from the process stream, it is important to have the best possfble estimates of inst.ument calibration for any fixed budget of time and effort. In principal, instruments can be calibrated to within the precision of preparation of laboratory standards, but in order to achieve this degree of precision 1t might be necessary to spend more time on the replication of calibration experiments than to the analysis of production samples. In this paper, we do not examine the question of how much effort should be spent on calibration experiments, but rather we develop methods of estimating callbration coefficients with the greatest precision possible from a fixed budget.

We consider only simple instruments that behave according to the following linear model:

$$
s_{t}=a_{t}+b_{t} q_{t}+\varepsilon_{0 t}
$$

where

$s_{t}=$ output sigal at time $t$

$a_{t}=$ instrument "zero" parameter

$b_{t}=$ instrument scale parameter

$q_{t}=$ true value of physical quantity of interest

$\varepsilon_{0 t}=$ random measurement error.

We assume that the instrument parameters are subject to a random walk type of drift. The evolution of parameters over time is given by 


$$
a_{t+1}=a_{t}+\varepsilon_{1 t}
$$

$$
b_{t+1}=b_{t}+\varepsilon_{2 t}
$$

where the random variables $\epsilon_{j t}$ have mean zero and are independent over time and between sequences, and have vartance $\sigma_{j}^{2}$, for $j=0,1,2$, .

The dynamic behavior of these parameters is a simple case of Equation (1.7) in which the state vector $Y_{t}$ has components $a_{t}$ and $b_{t}$ and the matrix $A$ is the identity matrix of order 2. The matrix $c_{v}$ is diagonal, containing the elements $\sigma_{1}^{2}$ and $\sigma_{2}^{2}$ on its diagonal.

Now suppose at time $t$ we perform the following calibration experiment: two standard solutions are prepared with properties (concentrations for example) having the values $q_{t}^{\prime}$ and $q_{t}^{\prime \prime}$, which are known within a range of uncertainty that is much smaller than the standard deviation of the measurement error. We assume that the calibration experiment using the two solutions is performed so quickly that the parameter drift during the experiment is negligible. For example, $t$ may be measured in days, while a calibration experiment may take less than an hour. In this case, the output signals obtained from the pair of standard solutions is given by Equation (2.1) which we write in matrix form as

$$
\left[\begin{array}{l}
s_{t}^{\prime} \\
s_{t}^{\prime \prime}
\end{array}\right]=\left[\begin{array}{ll}
1 & q_{t}^{\prime} \\
1 & q_{t}^{\prime}
\end{array}\right]\left[\begin{array}{l}
a_{t} \\
b_{t}
\end{array}\right]+\left[\begin{array}{c}
\varepsilon_{0 t}^{\prime} \\
\varepsilon_{0 t}^{\prime}
\end{array}\right] .
$$

Note that this equatior is in the form of (1.8) In contrast to many applications of Kalman theory, In this case the matrix $M$ in (1.8) is, in general, different each time the experiment is performed and is in fact determined by the choice of values of $q_{t}^{\prime \prime}$ and $q_{t}^{\prime}$. 
The Kalman recursive estimation scheme shows how to make the best use of the history of calibration experiments together with the latest observations in estimating the current values of the calibration coefficients. In order to apply the results of Section 1, one must know of the relative magnitudes of the error and drift variances. These may be estimated by performing groups of calibration experiments widely separated in time and using analysis of variance techniques based on the within group and among group variances.

It happens that, for this particular model, the covariance matrix of the estimated coefficients converges, as long as $q^{\prime}$ and $q^{\prime \prime}$ are not identical, to a limiting matrix that is independent of the covariance matrix of the initial estimate. Table 1 shows the computed limiting covariance matrices for several cases. In these computations, we fix the measurement etror varlance, $\sigma_{w}^{2}$, at 1 . To examine the effect of increasing the range of the calibration, we fix $q^{\prime}$ at the value 1 , and pick values of $\mathrm{q}^{\prime \prime}$ to be 3,10 or 100 . We assume that both the intercept and slope coefficlents are equally stable, that is that the two drift variances have the same value, $\sigma_{v}^{2}$. We consider a relatively stable Instrument, with $\sigma_{v}^{2}=.001$, and less stable instruments with $\sigma_{v}^{2}=.01$ and 0.1 . Taking all combinations, we carry out the computations for nine cases. 


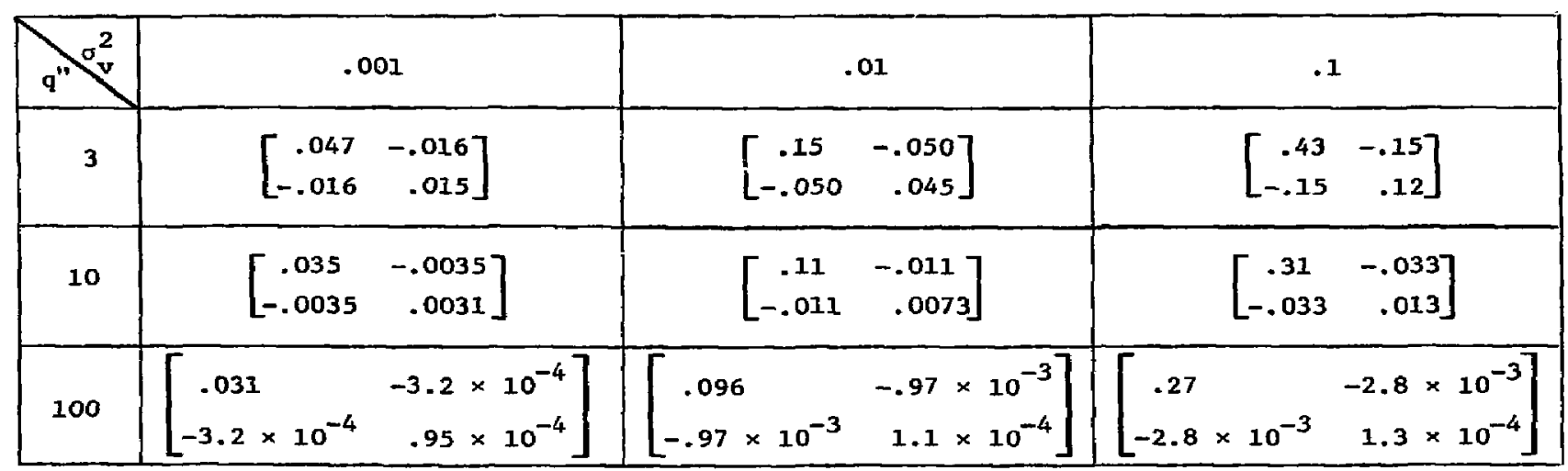

TABLE 1

LIMITING VARIANGE MATRIX OF INSTRUMENT PARAMETER ESTTMATORS

$$
\sigma_{w}^{2}=1, M=\left[\begin{array}{ll}
1 & 1 \\
1 & q
\end{array},\right]
$$


As can be seen from Table 1, increasing the baseline length reduces all elements in the variance matrix of the estimators for each instrument; the variance of the estimate of slope is reduced more than the variance of estimated intercept. If the instrument is known to be truly linear, the best choice of callb' stion values is at the extreme ends of the instrument operating range. In practice, of course, it is often desirable to check calibration at the midrange as well to test the hypothesis of linearity. Clearly, the less stable instrument has a larger variance of estimated calibration coefficients. As mentioned earlier, the variance of the estimated coefficients can be reduced by repilcating the calibration experiment, which has the effect of reducing the vartance of the measurement error $\sigma_{\mathrm{w}}^{2}$.

If both $\sigma_{\mathrm{w}}^{2}$ and $\sigma_{\mathrm{v}}^{2}$ are multiplied by the same scale factor, so is the variance matrix of the estimated coefficients. If, for example, $\sigma_{v}^{2}=.001$ and $\sigma_{w}^{2}=, 1$ the ratio is .01 , hence the vartance matrix is obtained by multiplying the middle colum of Table 1 by 0.1 . 


\section{RETROSPECTIVE UPDATE}

Observations obtained after time $t$ may be used to improve our estimate of the system state at $t$. In this section, we consider $t$ fixed and denote future epochs by $t+k$, where $k>0$. Even though we are primarily interested in estimating $Y_{t}$, we continue to estimate $Y_{t+k}$ for $k=1,2, \ldots$ by the scheme described in Section 1. Suppose that the history of observations to time $t+k$ has been used to estimate both $Y_{t}$ and $Y_{t+k}$, that 1s, both $\hat{Y}_{t \mid t+k}$ and $\hat{Y}_{\tau+k \mid t+k}$ have been computed. Then we observe the next output vector $x_{t+1+1}$ and desire to update our estimate of $Y_{t}$.

As in Section 1, this estimate is updated by using

$$
\hat{Y}_{t \mid t+k+1}=\hat{Y}_{t \mid t+k}+B_{t \mid t+k+1}{ }^{2} t+k+1
$$

where B is computed, as In Equation (1.3), from

$$
B_{t \mid t+k+1}=C\left[R_{t \mid t+k}, z_{t+k+1}\right\} C\left[z_{t+k+1}\right]^{-1}
$$

and the variance matrix of the residual of the updated estimator is obtained, as in Equation (1.5), irom

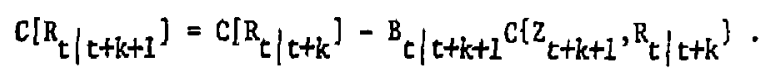

In order to carry out these calculations, we need to compute the matrix $C\left\{z_{t+k+1}, R_{t} \mid t+k\right\}$. Observe first that

$$
z_{t+k+1}=M A R_{t+k \mid t+k}+M V_{t+k}+W_{t+k+1}
$$


Since $R_{t \mid t+k}$ 1B a linear combination of the $V_{j}$ sequence for $j<t+k$ and the $w_{j}$ sequence for $j<t+k+1$, it is uncorralated with $v_{i+k}$ and $\mathrm{H}_{t+k+1}$. Hence we have

$$
C\left\{z_{t+k+1}, R_{t} \mid t+k\right\}=\operatorname{MAC}\left\{R_{t+k \mid t+k}, R_{t} \mid t+k\right\} \text {. }
$$

Now notice that

$$
R_{t \mid t+k}=R_{t \mid t}-\sum_{j=1}^{k} B_{t \mid t+j}{ }^{2} t+j
$$

Since $R_{t+k \mid t+k}$ 1s uncorrelated with $z_{t+j}$ for $j<k+1$ as shown by Equation $(1.20)$, we have

$$
C\left\{R_{t+k \mid t+k}, R_{t \mid t+k}\right\}=c\left\{R_{t+k}\left|t+k, R_{t}\right| t\right\}
$$

Finally we show how to update the term in the right of (3.7). Using Equation (1.19) to deduce the relationshlp between $R_{t+k \mid t+k}$ and $R_{t+k+1 \mid t+k+1}$, we have

(3.8) $C\left\{R_{t+k+1 \mid t+k+1}, R_{t \mid t}\right\}=\left(I-B_{t+k+1 \mid t+k+1} M\right) A C\left\{R_{t+k \mid t+k}, R_{t} t^{\}}\right.$.

In summary, Equations (3.1), (3.2), and (3.5) perinits update of the estimate $\hat{Y}_{t \mid t+k}$. The variance of this estinator is updated recursively using (3.3), which also requires (3.5). This Equation, in turn, depends on (3.7) and the recursion of (3.8). The matrices $c\left[2_{t+k+1}\right]$ in (3.2) and $B_{t+k+1 \mid t+k+1}$ in (3.8) are obtained from the ordinary Kalman recursion of Section 1 . 


\section{APPLICATION OF RETROSPECTIVE UPDATE TO INSTRUMENT CALIBRATION}

In order to fllustrate the use of the methods of the previous section, the instrument model of Section 2 has been studied in some detail. In these computations, we consider the four extreme cases of Table 1, that is, $\sigma_{v}^{2}=.1$ and .001 and $q^{\prime \prime}=3$ and 100 . The covariance matrix $C\left[R_{t} \mid t+k\right]$ was computed for values of $k$ ranging from 1 to 128 , and the main dlagonal alements plotted against $k$, with a logarithmic transformation of the $k$ axis. Recall that the first component of the state vector in this model is $a_{t}$, the intercept of the calibration curve, and the second is the slope, $b_{t}$. The graphs of Figures 1-4 show the variances of estimates of these quantities.

It is clear from these plots that the maximum reduction in the variance of $\hat{b}_{t}$ is considerably less than for $\hat{a}_{t}$, particularly when $q^{\prime \prime}$ is set to 100 in the long base line case. In this case, no significant improvement Is possible because each individual calibration experiment yields essentially all the information about the current value of slope, $\hat{b}_{t}$, that can be obtained. On the other hand, the variance of the estimate $\hat{a}_{t}$ can be reduced by about. a third by future calibration experiments. We observe that when the instrument is unstable, that is $\sigma_{\mathrm{v}}^{2}$ is large compared to $\sigma_{\mathrm{w}}^{2}$, the distant future contains little information about current values of the intercept $a_{t}$. The values of $\sigma_{\hat{a}}^{2}$ declines to its limit fairly rapidly; after 8 perlods have elapsed, further observations do not improve the estimate. By contrast, the more stable Instrument shows a decline in $\sigma_{\hat{a}}^{2}$ for 64 or 128 additional observations, but the inftial decrease is not so rapid, After the first 8 additional observations, the variance has decreased by less than a third of the ultimate amount. 


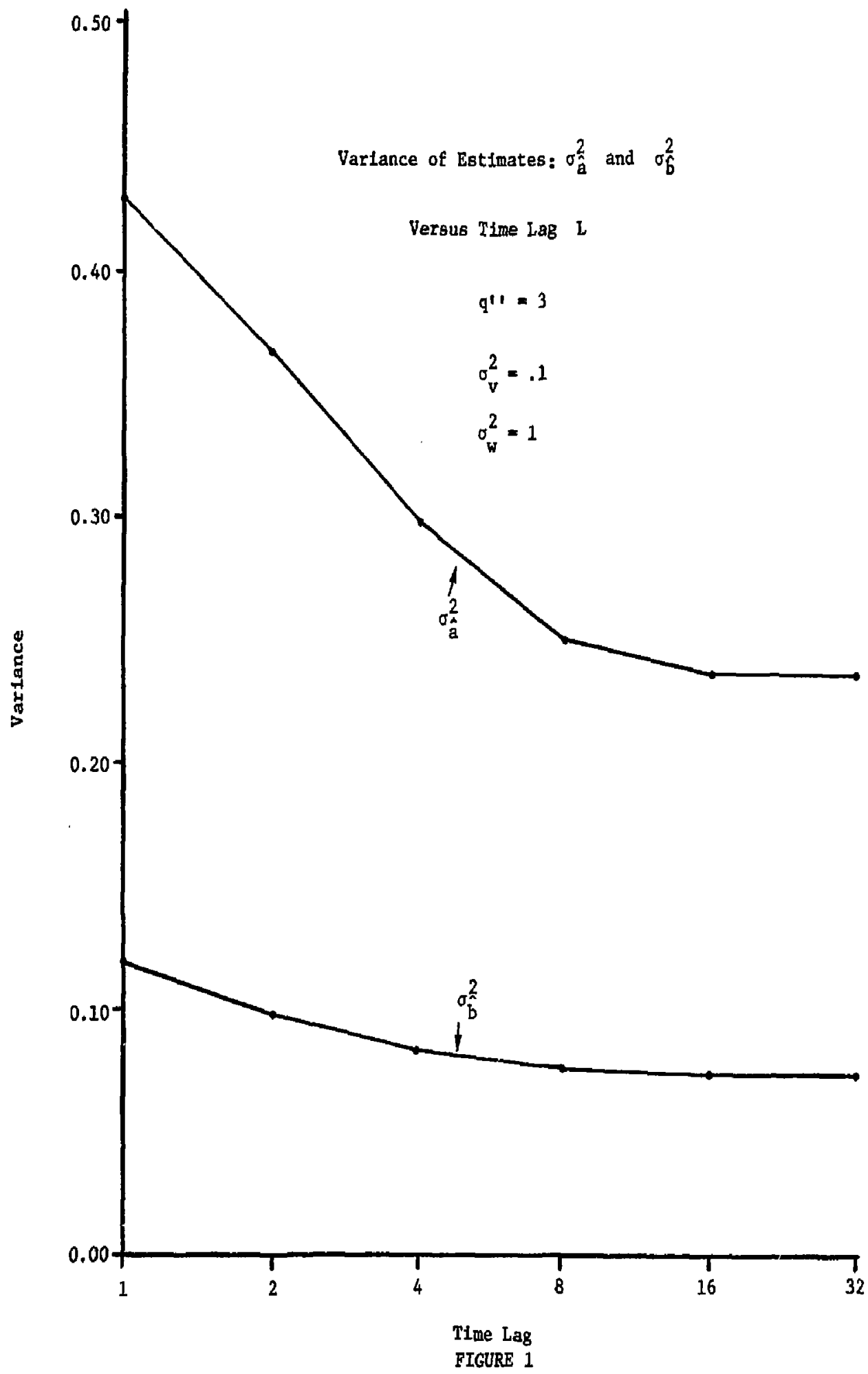




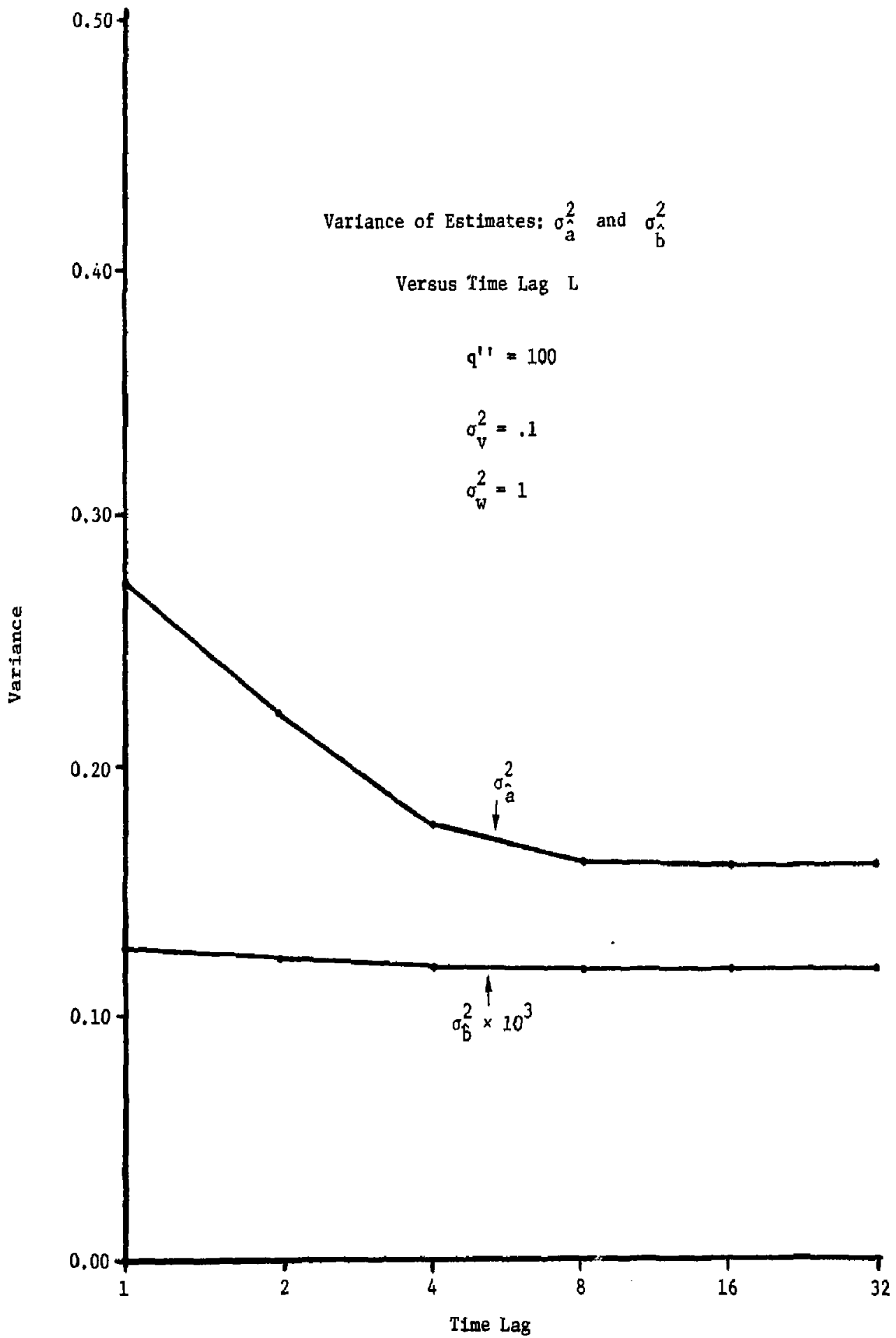

FIGURE 2 


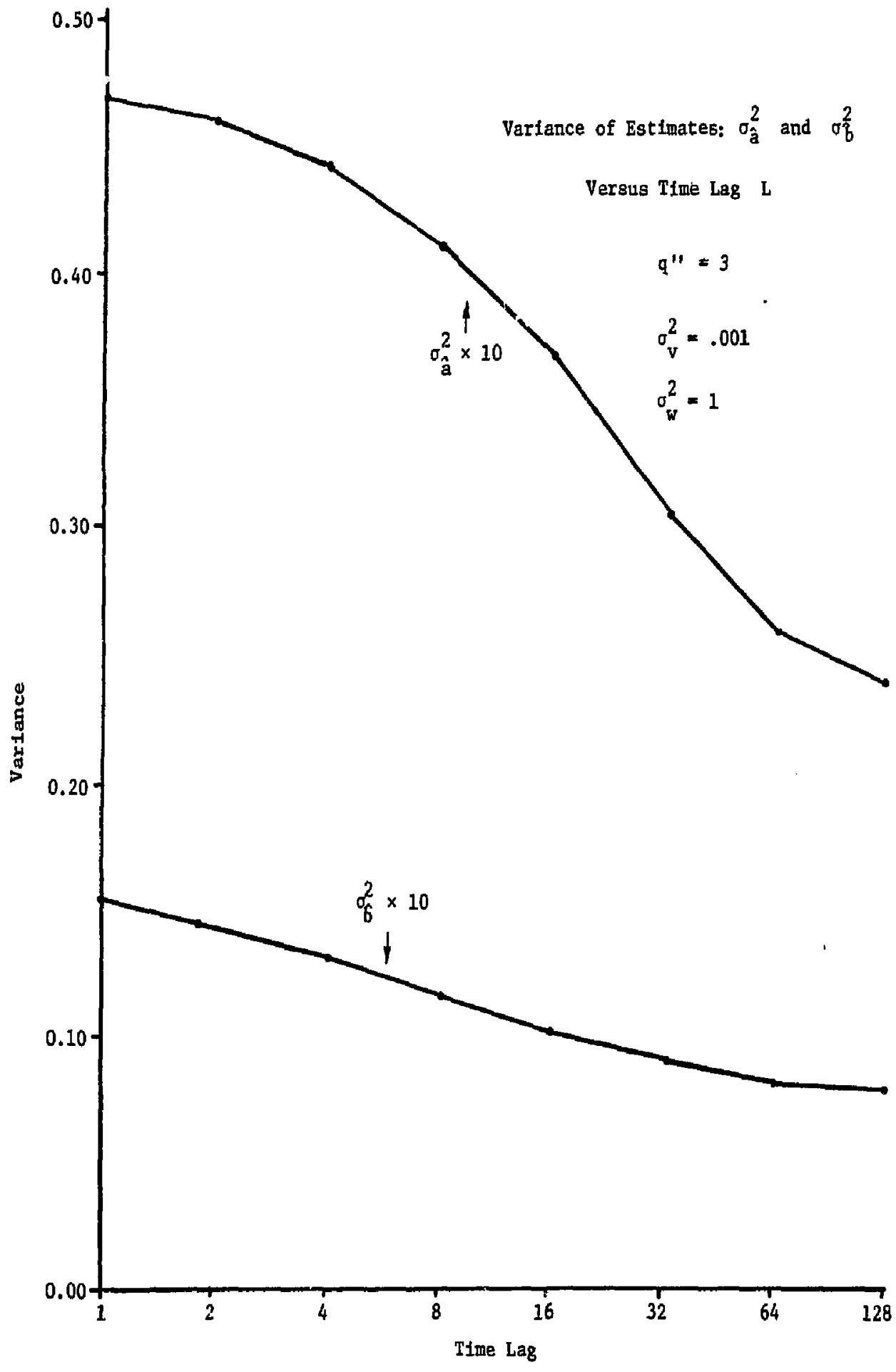

FIGURE 3 


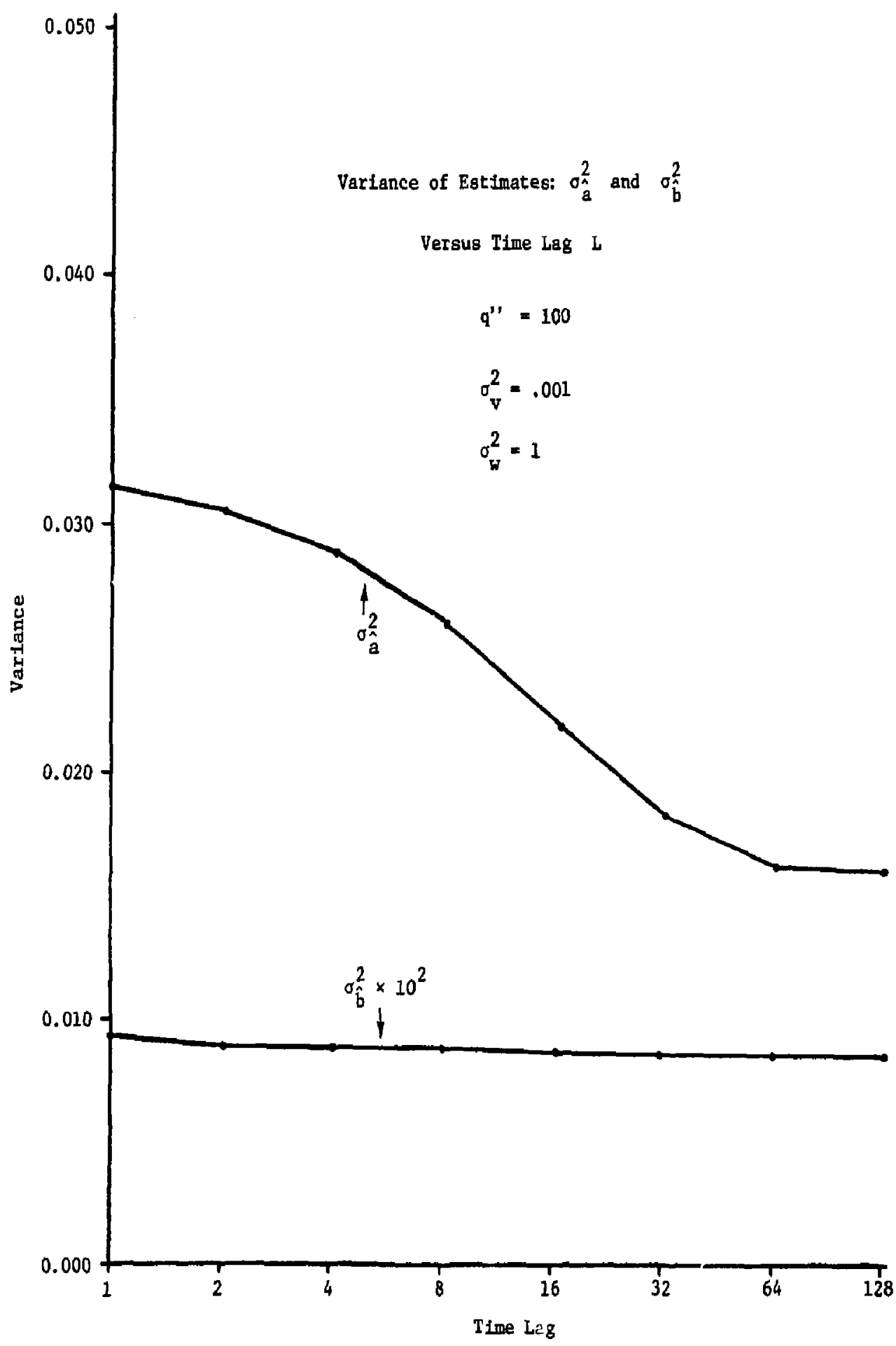

FIGURE 4 
Table 2 shows the comparison between the variance matrices of the estimators before retrospective update and after. The flrst matrix in each block is copied from Table 1. The second is the result after improvements from retrospective updating become insignificant. The diagonal elements of each matrix . e the variances of $\hat{a}$ and $\hat{b}$ respectively, and the off diagonal ele snt is the covariance of $\hat{a}$ with $\hat{b}$.

We conclude from these examples that, for xelatively unstable Instruments, the retrospective update scheme may be an attractive way to Improve the precision of estimated calibration coefficients since a few additional observations will yield significant variance reduction. However, if the instrument is relatively stable, many more additional calibration experiments must be performed to gain significant varlance reduction. Furthermore, the variance of the estimated coefficients produced by the usual Kalman filter scheme is smaller for a more stable instrument, other things being equal, so the retrospectlve update of calibration coefficlents may not be worthwhle in thls case.

Since scaling all input variances by a constant multiplies the estimator varlances by the same constant, as has been mentioned earlier, the stability of the instrument depends on the ration of $\sigma_{\mathrm{W}}^{2}$ to $\sigma_{v}^{2}$ rather than the absolute value of $\sigma_{w}^{2}$. The effective value of $\sigma_{w}^{2}$, the measurement error variance, can be reduced by replicating the experimental design and averaging the estimates is derived from each independent replicate. The result, of course, would be to reduce the variance of the retrospectively updated estimates. Since the ratio of $\sigma_{w}^{2}$ to $\sigma_{v}^{2}$ would be effectively reduced, so would relative stability. Consequently, the additional rariance reduction obtained from retrospective updates will occur sooner, which would make the retrospective update scheme even more attractive. 


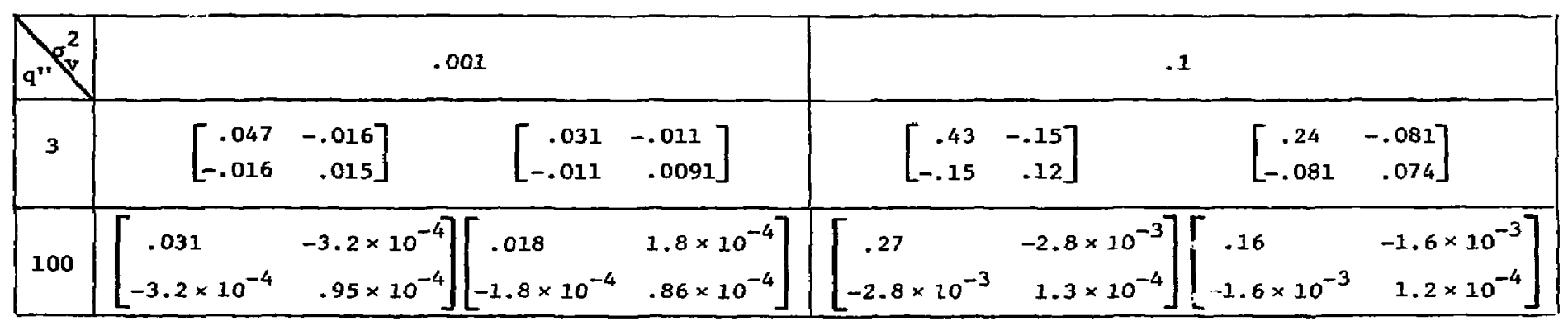

TABLE 2

COMPARISON BETWEEN LIMITING VARIANCE MATRIX AND

UPDATED VARIANCE MATRIX OF INSTRLMENT PARAMETER ES TMATORS

$$
\sigma_{w}^{2}=1, M=\left[\begin{array}{ll}
1 & 1 \\
1 & q
\end{array}\right]
$$




\section{INTERPOLATION COMBINED WITH RETROSPECTIVE UPDATE}

In this section, we assume that observations are made at every $k$ periods, that is when $t=$ mk . All the observations obtained until epoch mk are used to find the best linear unblased estimators of the system states at times $t=n k$, denoted $\hat{\mathrm{Y}}_{\mathrm{nk} \mid \mathrm{mk}}$, for $n=1,2, \ldots, \mathrm{m}$. The same information is used to estimate the system states of the periods between observation epochs 1.e., $\hat{Y}_{\text {nkt }+\ell \text { mk }}, \ell=1,2, \ldots, k-1$; $\mathrm{n}=1,2, \ldots, \mathrm{m}-1$.

The new observation vector $x_{(m+1) k}$ may be used to update our estimates $\hat{\mathrm{Y}}_{\mathrm{nk}+\ell \mid \mathbb{k} \times}$ 1.e., to find the improved estimates $\hat{\mathrm{Y}}_{\mathrm{nk}+\ell \mid(m+1) k}$, $a=1,2, \ldots, m ; \ell=1,2, \ldots, k$ and also the new estimates $\hat{y}_{m k+\ell \mid(m+1) k}$, $\ell=1,2, \ldots, k$, of the system state at times between the latest observation and the previous one.

\subsection{Update of Estimate at Observation Epochs}

The recursive estimation of system state at the observation epochs $t=m k, m=1,2, \ldots$, accomplished by methods directly analogous to the basic Kalman recursion of Section 1.

Applying the Ilnear dynamic relation Equation (1.7) $k$ times recursively, we have

$$
Y_{(m+1) k}=A^{k} Y_{m k}+V_{m k}^{* k}
$$

where we define

$$
v_{m k}^{* \ell}=A^{l-1} v_{m k}+A^{l-2} v_{m k+1}+\ldots+v_{m k+l-1}
$$

and hence

$$
C\left[v^{* \ell}\right]=C\left\{v_{m k}^{* \ell}, v_{m k}^{* \ell}\right\}=\sum_{j=0}^{\ell-1} A^{j} C_{v}\left(A^{j}\right)^{\prime}
$$


thus,

$$
\hat{\mathrm{T}}_{(m+1) \mathrm{k} \mid m k}=\mathrm{A}^{\mathrm{k}_{\mathrm{Y}}} \mathrm{mk} \mid \mathrm{mk} \text {. }
$$

We obtain from the above two equations, and the definition of the residual vector $R$,

$$
R_{(m+1) k \mid m k}=A^{k} k_{m k \mid m k}+V_{m k}^{* k} \text {. }
$$

Now as in Section 1 , we have

$$
z_{(m+1) k}=x_{(m+1) k}-\hat{x}_{(m+1) k \mid m k}
$$

$$
\begin{aligned}
& =\mathbb{M R}_{(m+1) k \mid m k}+W_{(m+1) k} \\
& =M\left(A^{k_{R k} \mid m k}+V_{m k}^{* k}\right)+W_{(m+1) k} .
\end{aligned}
$$

The estimate of $Y_{(m+1) k}$ is updated from time mk by

$$
\hat{\mathrm{Y}}_{(m+1) k \mid(m+1) k}=\mathrm{A}^{\mathrm{k}} \hat{\mathrm{Y}}_{m k \mid m k}+\mathrm{B}_{(m+1) k \mid(m+1) k^{2}(m+1) k}
$$

where the matrix $B$ is computed from

$$
{ }_{(m+1) k \mid(m+1) k}=C[R(m+1) k \mid m k) M^{\prime} C\left[z_{(m+1) k}\right]^{-1} \text {. }
$$

The covariance matrices of the residual of the observation is computed using Equation (5.4b) from

$$
C\left[Z_{(m+1) k}\right]=\operatorname{MC}\left[R_{(m+1) k \mid m k}\right] M^{\prime}+C_{w} \text {. }
$$

The variance matrix of the updated state vector estimate is

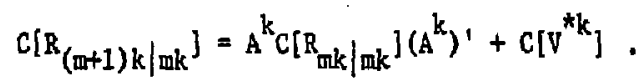


Notice that, in the procedure for computing $\hat{Y}_{(m+1) k} \mid(m+1) k$ we

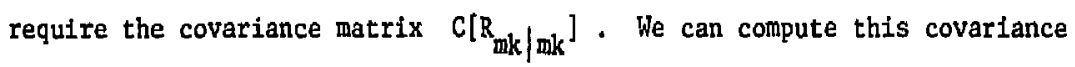
matrix recursively from Equations $(5.6),(5.7)$ and (5.8) in the usual way.

$$
C\left[R_{(m+1) k \mid(m+1) k}\right]=\mathcal{C}\left[R_{(m+1) k \mid m k}\right]-B_{(m+1) k \mid(m+1) k}
$$

$$
\text { - } C\left\{z_{(m+1) k}, R_{(m+1) k \mid \text { ink }}\right\} \text {. }
$$

\subsection{Interpolation}

Next we show how to estimate the system states of the periods between observation epochs and compute the corresponding covariance matrix of residuals. To extrapolate from the latest observation into the future, we use the analog of Equation (5.1):

$$
\hat{\mathrm{Y}}_{\mathrm{mk}+\ell \mid m k}=\mathrm{A}_{\mathrm{mk} \mid \mathrm{mk}}^{\ell}, \ell=1,2, \ldots, \mathrm{k} .
$$

Interpolation and retrospective update are accomplished by:

$$
\hat{Y}_{n k+l \mid m k}=\hat{Y}_{n k+l \mid(m-1) k}+B_{n k+l \mid m k} Z_{m k}, n=m-1, m-2, \ldots
$$

where

$$
\mathrm{B}_{\mathrm{nk}+\ell \mid \mathrm{mk}}=\mathrm{C}\left\{\mathrm{R}_{\mathrm{nk}+l \mid(\mathrm{m}-1) \mathrm{k}}, \mathrm{Z}_{\mathrm{mk}}\right\} \mathrm{C}\left[\mathrm{Z}_{\mathrm{mk}}\right]^{-1}
$$

The matrix $\mathrm{C}\left[\mathrm{z}_{\mathrm{mk}}\right]^{-1}$ is obtained using the Equation (5.7). We show how to compute $C\left\{R_{n k+l} \mid(m-1) k, Z_{m k}\right\}$ by recursion on $\ell$ first, then by recursion on $m$.

We focus our attention on the interval $t=n k, n k+1, \ldots, n k+\ell, \ldots$, $(n+1) k$, which includes the two observation epochs nk and $(n+1) k$. We consider first the Interpolation between the latest two observation epochs, that is, the latest observation is $x_{m k}$ where $m=n+1$. Then we show how to carry out the retrospective update computation as additional observations become avallable, that 1s when $m>n+1$. 


\subsubsection{Interpolation When $n=m-1$}

$$
\text { Cons1der III }=n+1 \text {. S1nce } R_{n k+\ell+1 \mid n k}=A_{n k+\ell / n k}+V_{n k+l} \text {, }
$$

and using Equation $(5.4 \mathrm{c})$, we have the recursion on $\ell$ :

(5.11a) $\quad C\left\{R_{n k+l+1 \mid n k}, Z_{(n+1) k}\right\}=A C\left[R_{n k+l \mid n k}, Z_{(n+1) k}\right\}+C_{v}\left(A^{k-l}\right) M^{\prime}$ and the initialization:

$$
\left.C R_{n k \mid n k}, Z_{(n+1) k}\right]=C\left[R_{n k \mid n k}\right]\left(A^{k}\right)^{\prime} M^{\prime}
$$

These matrices are used in Equation (5.10) to compute $\hat{\mathrm{Y}}_{\mathrm{nk}+l} \mid(\mathrm{n}+1) k$.

\subsubsection{Retrospective Update of Interpolation: $\mathrm{m}>n+1$}

Now we consider subsequent observations when $m>n+1$, i,e., $m=n+2, n+3, \ldots$. Utilization of $(5.10 c)$ requires computation of $C\left\{R_{n k+l \mid(m-1)}, Z_{m k}\right\}$. From the definftion,

$$
C\left\{R_{n k+l \mid(m-1) k} Z_{m k}\right\}=E\left\{\left(Y_{n k+\ell}-\hat{Y}_{n k+l \mid(m-1) k}\right)\left(Z_{m k}\right)^{\prime}\right\} .
$$

Now notice that,

$$
Y_{n k+\ell}=A^{l} Y_{n k}+V_{n k}^{* l}
$$

and

$$
\hat{Y}_{n k+l \mid(m-1) k}=\sum_{j=n+1}^{m-1} B_{n k+l \mid j k}{ }^{z}+A^{\ell} \hat{Y}_{n k} \mid n k
$$

from recursive substitution in Equation $(5.10 \mathrm{~b})$. By application of Equation (1.20),

$$
\mathrm{C}\left\{\mathrm{R}_{\mathrm{nk} \mid \mathrm{nk}}, 2_{\mathrm{mk}}\right\}=0, \pi=1,2,3, \ldots \mathrm{m} .
$$


Expanding the Equation (5.12) using the results of (5.4), (5.13) and (5.14), setting to zero the cross product terms which are uncorrelated and utilizing (5.15), we obtain:

$$
\mathrm{C}\left\{\mathrm{R}_{\mathrm{nk}+\ell} \mid(\mathrm{m}-1) \mathrm{k}, \mathrm{Z}_{\mathrm{mk}}\right\}=M A^{\mathrm{k}}\left[\mathrm{C}\left\{\mathrm{R}_{(\mathrm{m}-1) \mathrm{k} \mid(\mathrm{m}-1) \mathrm{k}}, \mathrm{R}_{\mathrm{nk} \mid \mathrm{nk}}\right\}\left(\mathrm{A}^{\ell}\right)^{\prime}\right.
$$

$$
\left.\left.+c\{R(m-1) k](m-1) k \cdot v_{n k}^{* l}\right\}\right]
$$

We derive the following recursive formulation to apply to Equation (5.16).

$$
C\left\{R_{m k \mid m k}, R_{n k \mid n k}\right\}=\left(A^{k}-B_{m k \mid m k} M A^{k}\right) C\left[R_{(m-1) k}\left|(m-1) k^{, R}{ }_{n k}\right| n k\right\},
$$

$$
\mathrm{m}=\mathrm{n}+1, \mathrm{n}+2, \ldots
$$

(5.18a) $C\left\{R_{m k \mid m k}, V_{n k}^{* \ell}\right\}=\left(A^{k}-B_{m k \mid m k}^{M A}\right) C\left\{R(m-1) k \mid(m-1) k, V_{n k}^{* l}\right\}$,

(5.18b) $\quad C\left\{R(n+1) k \mid(n+1) k, v_{n k}^{* l}\right\}=\left(I-B_{(n+1) k} \mid(n+1) k\right)\left\{A^{k-1} C_{v}\left(A^{l}\right)+\ldots+A^{k-l} C_{v}\right\}$.

Notice that the matrix $B$ and $C\left[R_{n k \mid n k}\right]$ are known. Thus we use (5.17) and (5.18a) recursively with the initial equation (5.18b), then substitute to Equation (5.16) to compute the covariance matrix

$$
C\left\{R_{n k+l \mid(m-1) k},{ }_{m k}\right\}, m=n+2, n+3, \ldots .
$$

Finally we can compute the estimate using (5.10a) and (5.10b). 


\subsection{Summary}

In practice, as each new observation, $x_{(m+1) k}$ is obtained, the current state is estimated, and then the retrospective updates of estimated states at earlier epochs are computed. The computation proceeds as follows:

(1) Update $\hat{\mathrm{Y}}_{(m+1) \mathrm{k} \mid(m+1) \mathrm{k}}, \mathrm{C}\left[\mathrm{Z}_{(\mathrm{m}+1) \mathrm{k}}\right]$ and $\mathrm{C}\left[\mathrm{R}_{\left.(\mathrm{m}+1) \mathrm{k} \mid \mathrm{mk}_{\mathrm{k}}\right] \text { using }}\right.$ $(5.5),(5.6),(5.7)$ and $(5.8)$.

(2) Compute the interpolation estimates $\hat{\mathrm{Y}}_{\mathrm{mk}+l \mid(\mathrm{m}+1) \mathrm{k}}$ from (5.10b), (5.10c), (5.11a) and (5.11b). These yíelds also $c\left\{\left.R_{n k+\ell}\right|_{\text {ak }}\right.$ - $\left.Z_{(m+1) k}\right\}$, by recursion on $\ell$.

(3) Update earlier 1nterpolation estimates to compute $\hat{y}_{n k+l} \mid(m+1) k$ by use of $(5.10 b),(5.10 c)$ and applying $(5.16) \sim(5.18 b)$ for $\mathrm{n} \leq \mathrm{m}-1$. 


\section{INTERPOLAT'ION AND RETROSPECIIVE UPDATE OF INSTRUMENT CALIBRATION}

The computing scheme of Section 5 can be illustrated by a simpler version of the instrument model discussed in Sections 2 and 4, in which we assume that only a single parameter need be estimated. For example, with some instruments, a background reading is routinely taken with every production sample to determine the instrument "zero", so only the scale parameter need be determined by calibration. In this section, we let $\sigma_{\mathrm{W}}^{2}$ represent the variance of estimate of the current value of the instrument parameter as determined by a single calibration experiment. The variance of the parameter drift (per unit time) is $\sigma_{v}^{2}$ as before. In this case, of course, the instrument parameter and the observation resulting from a calibration experiment are scalar quantities.

We carry out the computations of Section 5 for instruments that are calibrated every 10 time units (days, for example). Two instruments are considered: a very stable instrument with $\sigma_{v}^{2}=.001, \sigma_{w}^{2}=1$; and a less stable instrument with $\sigma_{v}^{2}=.01$ and $\sigma_{w}^{2}=.1$. As before, scaling both input variances by a constant will scale the estimator variance by the same factor; it is the ratio $\sigma_{\mathrm{V}}^{2} / \sigma_{\mathrm{W}}^{2}$ that determines relative stability.

The behavior of the more stable instrument is shown in Figure 5, The top line of the plot shows the variance of the estimator $Y_{n k+l} \mid(n+1) k$ as a function of $\ell$. We assume that calibration experiments are performed every 10 days at times $t=10 n$ and $t=10 n+10$. We have computed the variance of the interpolated calibration coefficient estimates for the points between these epochs. The plot shows that the variance of the interpolated estimates lies on the straight line between the variances of the estimators for the epochs of calibration, represented by $\ell=0$ and $\ell=10$. The variance of the estimate 


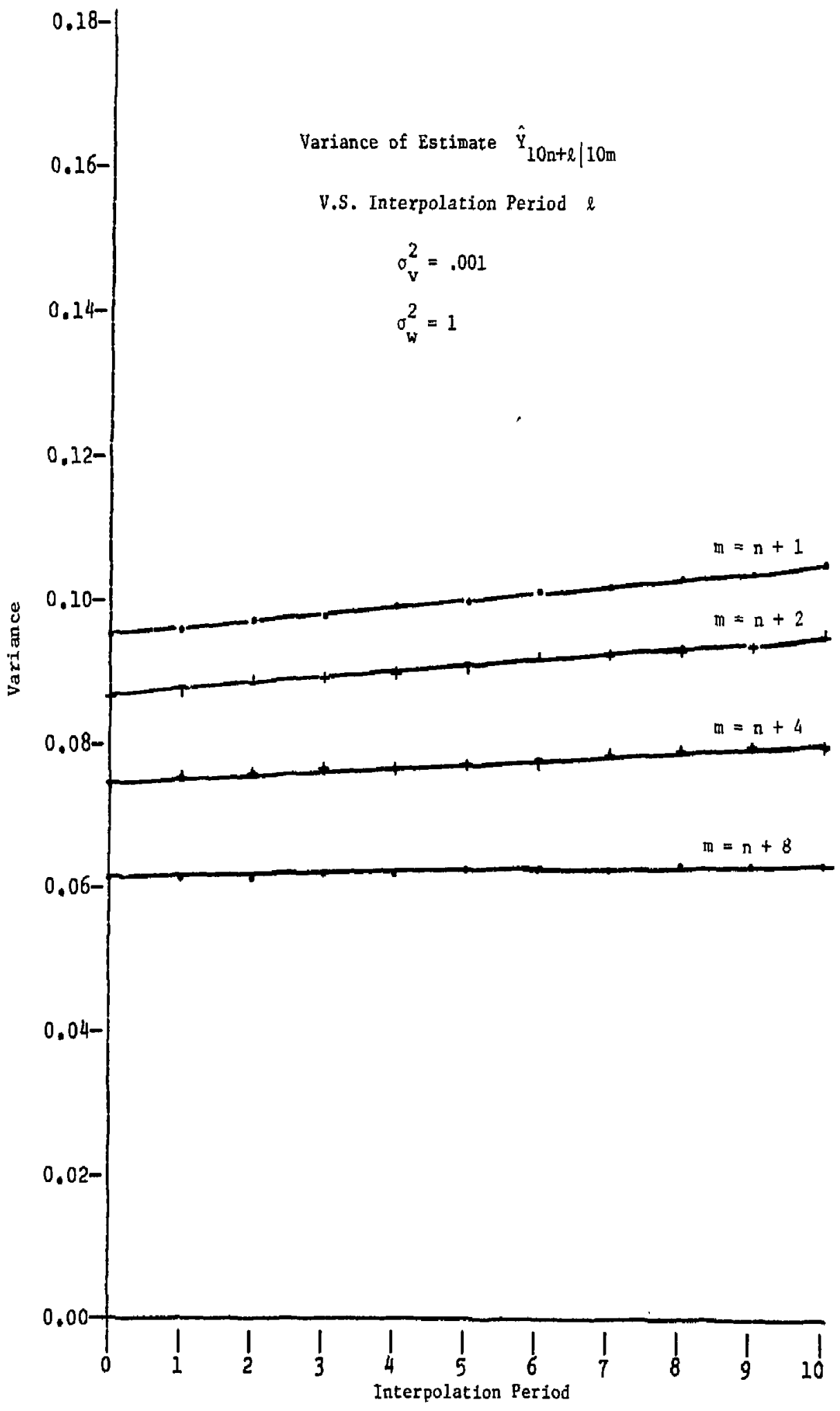


at $t=10 \mathrm{n}(l=0)$ 1s less than the variance at the next calibration epoch, $t=10 n+10 \quad(\ell=10)$ because the later calibration has provided information for the retrospective update of the earlier estimate.

The next calibration experiwent at $t=10 n+20$ is used to update the estimated states at both $t=10 n$ and $t=10 \pi+10$ and also the points between. The variances of these estimators fall on the line labeled "m $\times \mathfrak{n}+2 "$. Further retrospective updates of these estimators computed from calibrations performed at $t=10 n+40$ and $10 n+80$ give rise to variances that lie on the plotted lines labeled $m=n+4$ and $m=n+8$.

The time behavior of the varfances of the estimators at the calibration epochs and at the midpoint of the Interpolation Interval $(\ell=5)$ is shown on Figure 6. Here we abserve the typical pattern of stable instruments, that is relatively saall reduction in variance at first, but a continuing reduction as many additional observations become available. In this case, the variances of the estimates continue to decline out to the 16th additional data point at $t=10 n+160$.

By contrast, the corresponding plot for the less stable instrument, shown in Figure 8, exhibits a large variance reduction resulting from the first new calibration at $t=10 n+20$, and very small additional reductions. The variances of the estimated coefficients at all the interpolation points is shown In Figure 7. As with the stable instrument, the vartances of the first interpolated estimates lie in the straight line between the observation epoch variances. However, the plot of variances resulting from the first retrospective update at $t=10 n+20(m=n+2)$ assumes a pronounced curvature. 


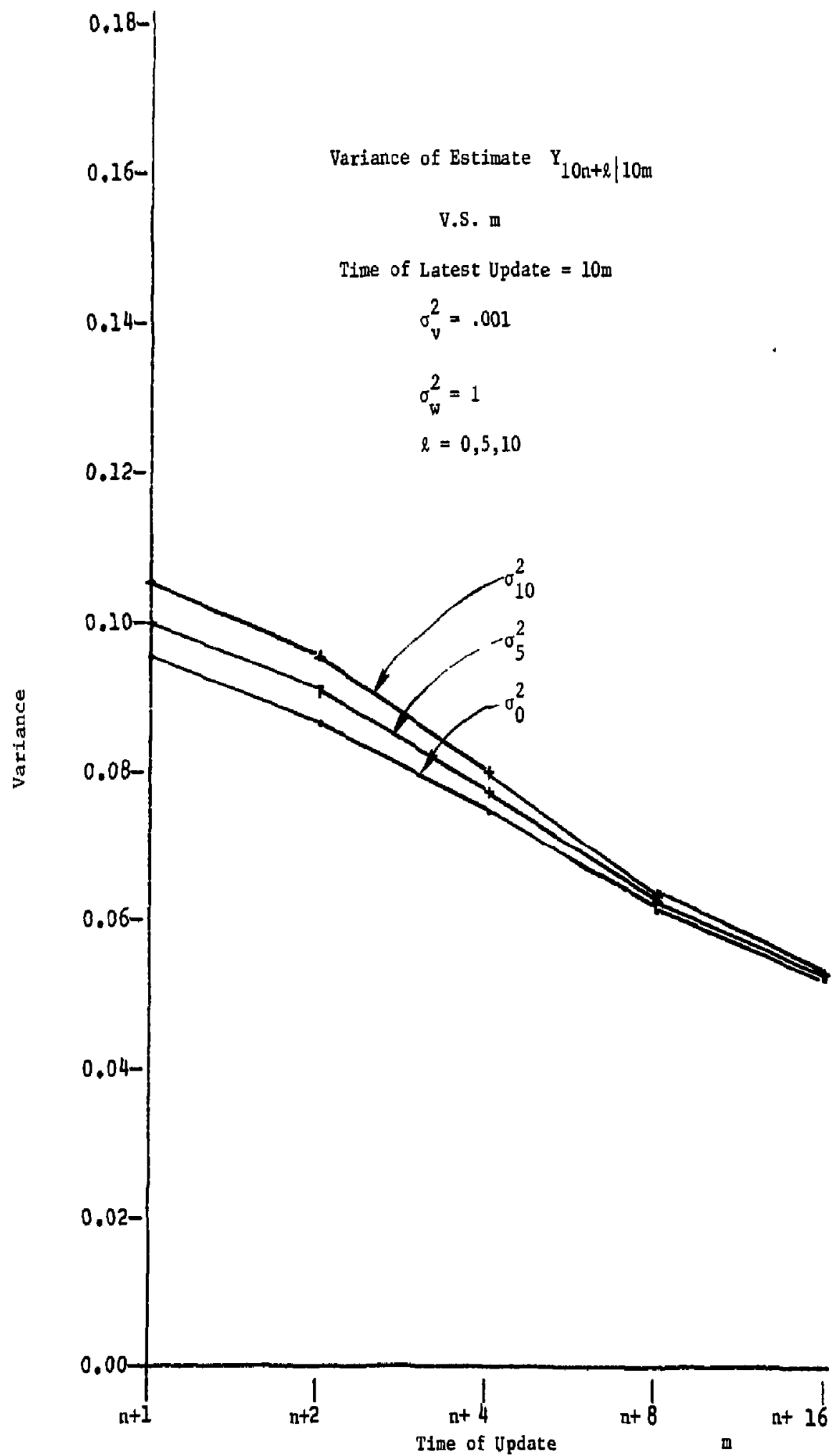

Is 
The plots for $m=n+4$ and $m=n+8$ essentlally coincide showing that more calibrations provide no additional information. The curvature of the lower plots shows that the calibration coefficlents are known with greater precision at observation epochs than at the points between.

In comparing the plots of Figure 6 and 8 , it is clear that the retrospective update of the interpolated estimates is much more attractive in the case of the less stable instrument because only one additional observation can result in substantial variance reductions (by factors as large as 2.5).

The theory o: Section 5 can be used to examine some questions of calibration strategy. Suppose, for example, each calibration experiment costs a dollar, and the budget for calibration is a dollar a day. Is it better to calibrate dafly or to perform 10 replicates every 10 daye? To be concrete, let us consider an instrument characterized by $\sigma_{v}^{2}=.0 \mathrm{t}$ and $\sigma_{w}^{2}=1$. If this instrument is calibrated daily and the retrospective update scheme is used, the variance of the estimate of the instrument parameter as a function of the time lag is shown in F1gure 9. Suppose that instead of the daily calibration, we replicate the calibration experiment 10 times every 10 th day and treat the average of 10 estimates as a single observation. Independent replicates will reduce the variance of this observation to $\sigma_{w}^{2}=.1$. Then the variance of the estimates derived from interpolation and retrospective update are shown in Figure 7 and 8 . Since we have assumed that the cost of 10 replicates is 10 times the cost of the basic calibration experiment, both strategles have the same cost.

The question of which strategy is to be preferred is a little complicated. If we do no retrospective updating, the 10 day strategy produces estimates with varlances ranging from .62 to 1.62 , while the dally calibration 
estimates have a variance of .95 . Suppose that one retrospective update is done in the 10 day scheme. Then the estimates have varlances shown in the middle line of Figure 7. Notice that these estimates range from 10 to 20 days old, the average age being 15 days. Now if we consult Figure 9, we see that estimates produced by daily scheme retrospectively updated for 15 days have a variance that is less than all but the first two points of the middle line of Figure 7. The varlance ratios of the largest and smallest vartances on that line to the 15 day point of Figure 9 and 1.2 and .9 . Thus when the time lags Involved in the retrospective update are comparable, the daily calibration scheme produces slightly more precise estimates than the 10 day scheme.

Under our assumption that each calibration experiment costs a dollar, it is slightly preferable to calibrate as often as the budget will allow rather than allowing more time to elapse and replicating the calibration experiments. However, if this assumption about costs fall to hold, a different conclusion follows, For example suppose the cost of two replicates performed sequentially is less than twice that of a single experiment. Then a flxed budget can be more effectively spent on less frequent replicated calibration experiments with retrospective updating of the interpolated estimates. 
$0.18-\mid$ Variance of Estimate $Y_{10 \mathrm{n}+\ell \mid 10 \mathrm{~m}}$

V.S. Interpolation Perfod $\ell$

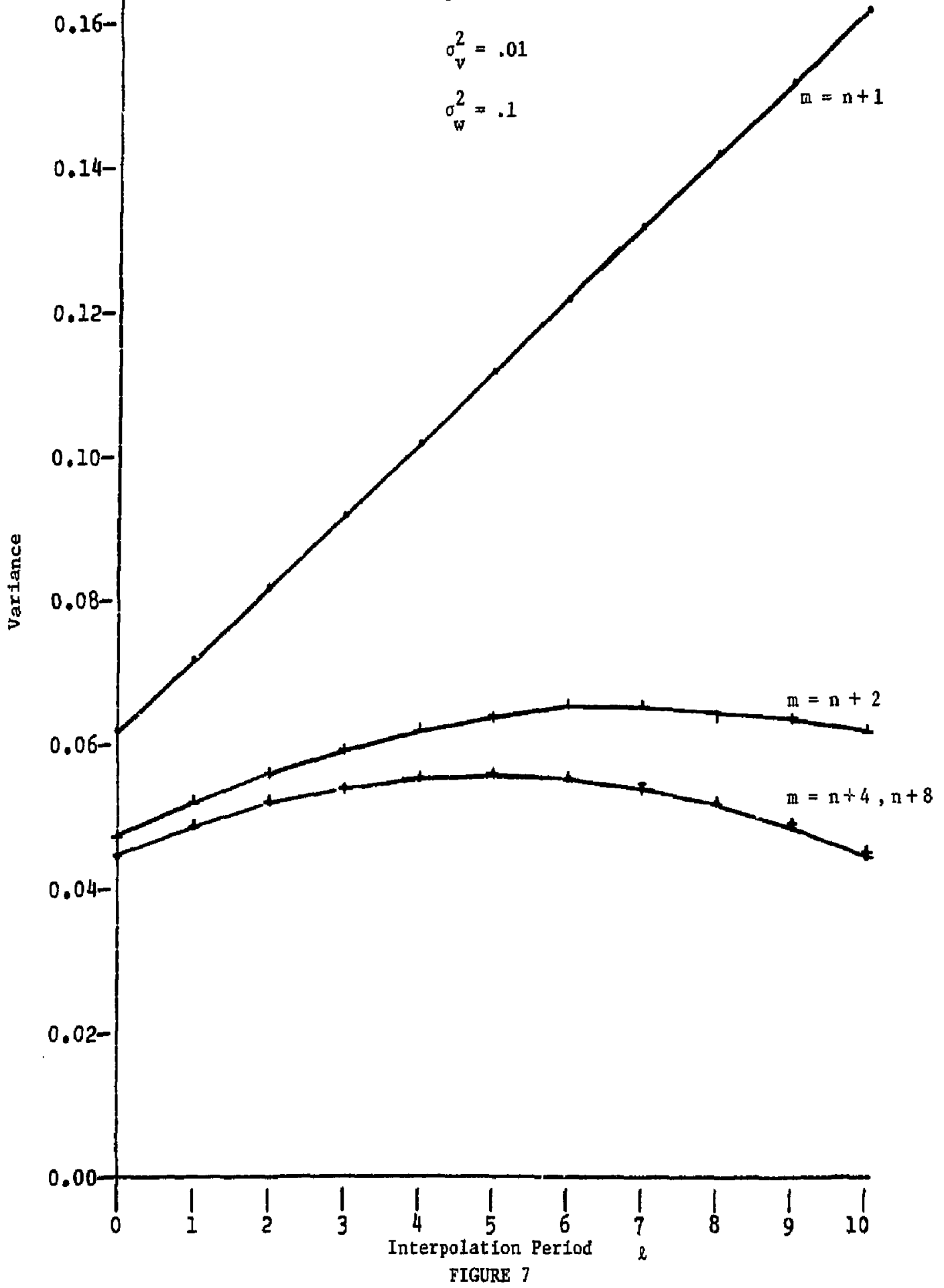




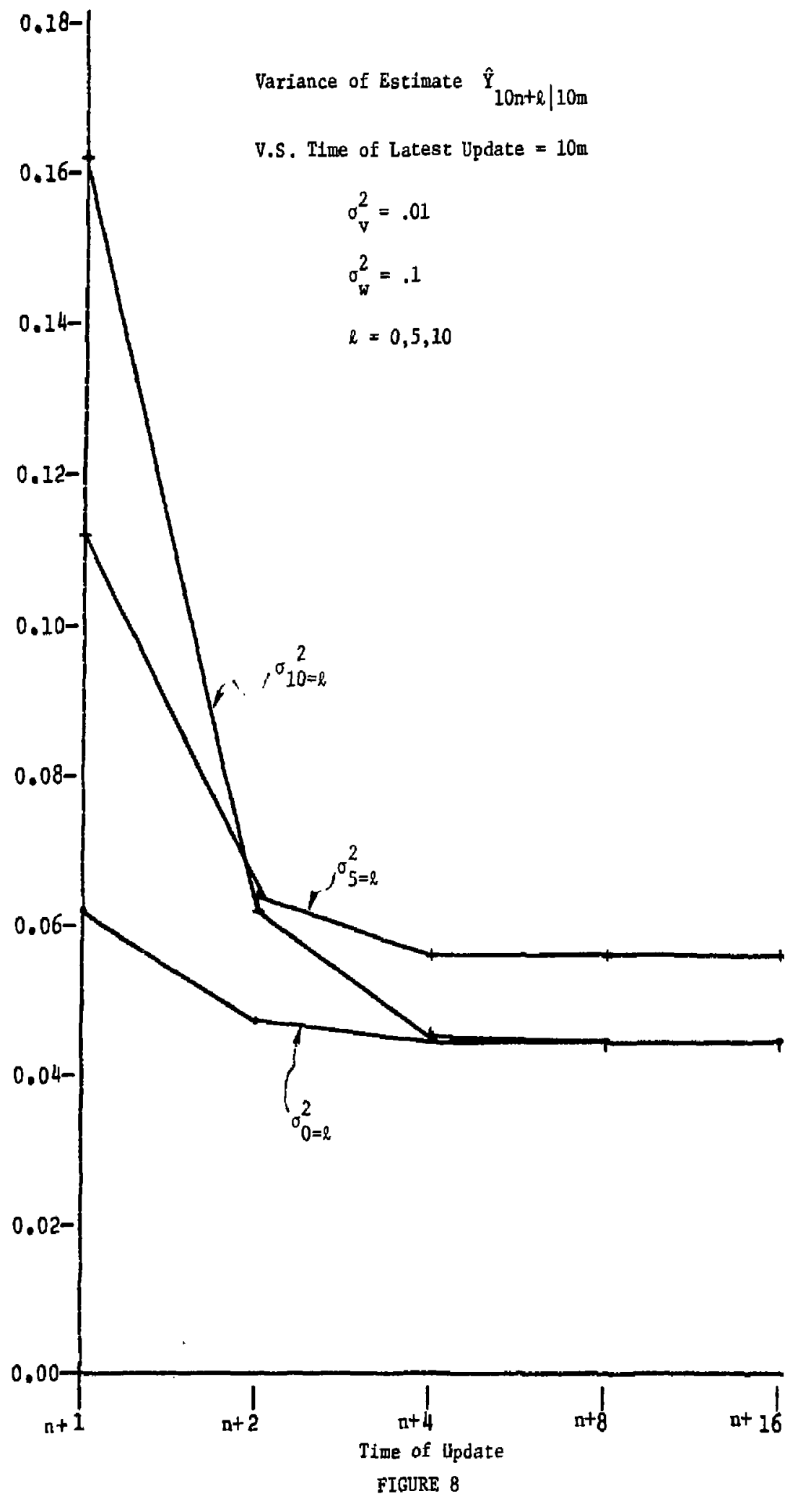



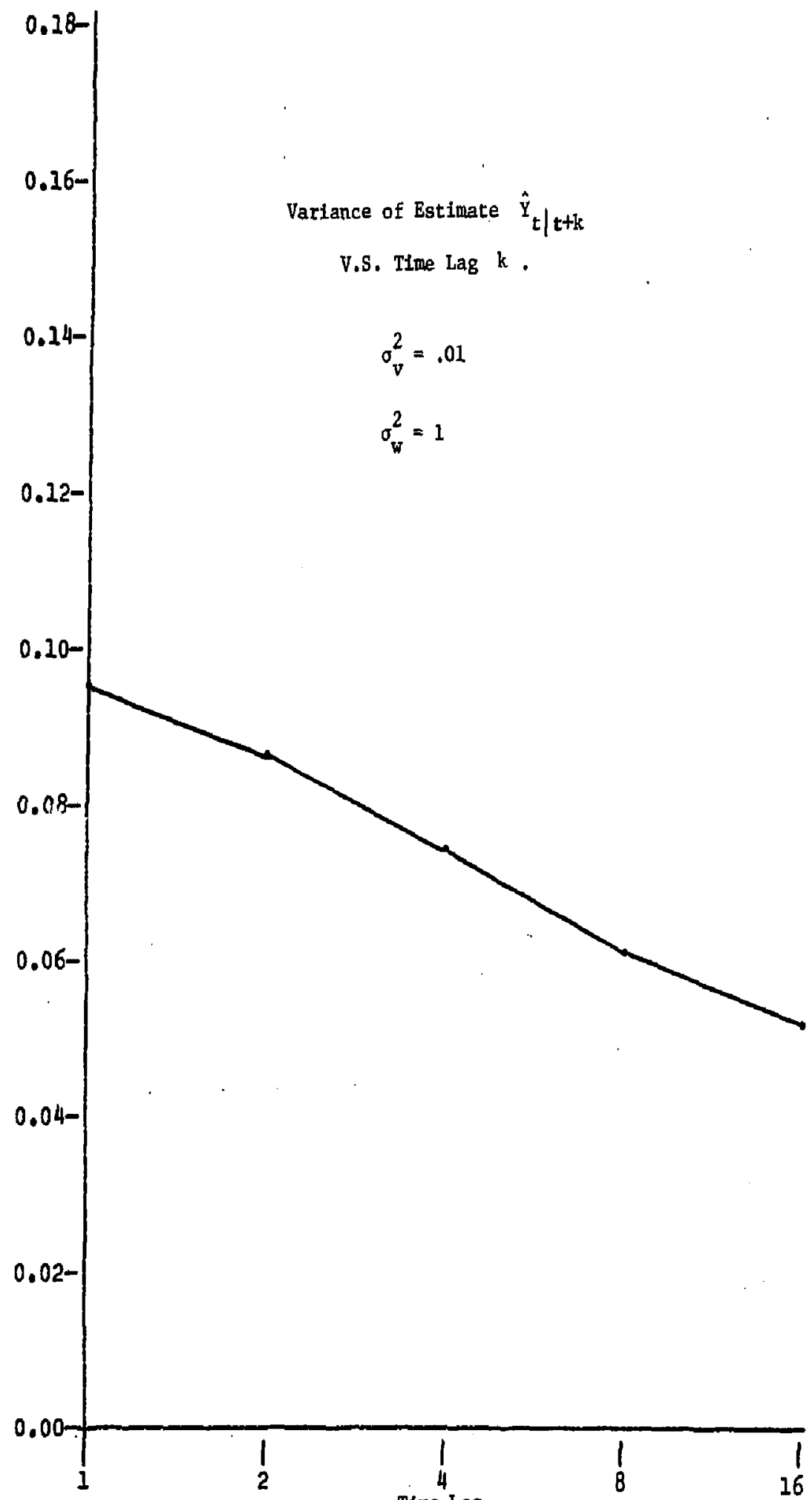

Tine Lag

FIGURE 9 


\section{REFERENCES}

[1] Kalman, R. E., "A New Approach to Linear Filtering and Prediction Problems," Trans. ASWE, J, Basic Eng., Vo1. 82, pp. 35-44 (1960).

[2] Kalman, R. E. and Bucy, "New Results in Linear Filtering and Prediction Theory," Trans. ASME, J. Basic Eng., Vo1. 83, pp. 95-108, (1961).

[3] Gelb, A., Applied Optimal Estimation, M.I.T. Press, Cambridge, Massachusetts, (1974).

[4] Jaech, J, L., Statistical Methods in Nuclear Material Control, U.S. Atomic Energy Commission, (1973).

[5] Railath, T., "An Innovations Approach to Least-Squares Estimation Part I: Linear Filtering in Additive White Noise," IEEE Trans. Aut. Con., Vol. AC-13, Pp. 646-655, (December 1968).

[6] Box, G. E. P., and G. M. Jenkins, Time Series Analysis: Forecasting and Control, Holden-Day, (1976).

[7] Sorenson, B. W., "Kalman Filtering Techniques," Advances in Control Systems, Vo1. 3, Academic Press, (1966).

[8] Fagin, S. L., "Recursive Linear Regression Theory, Optimal Filter Theory and Error Analyses of Optimal Systems," 1964 IEEE Conv. Rec., pp. 216-240. 


\section{NOTICE}

"This report was prepared as an account of work sponsored by the United Stales Government. Neither the Uniled States nor the United States Department of Energy, nor any of their employees. nor any of their contrastors, subcontractors, or their employess, makes any warranty, express or implied, or assumes any legal liability or responsibility for the accuracy, completeness or usefulness of any information, apparatus, product or process disclosed, or represents that its use would nol isfringe ssivatiby-sented rights." 